\title{
DESARROLLO, IMPLEMENTACIÓN Y BENEFICIOS DEL SISTEMA DE ASESORAMIENTO AL REGANTE - SAI EN BRASIL
}

\author{
Silva do Nascimento, A. K. ${ }^{1}$, Tarjuelo Martín-Benito, J. M. ${ }^{2}$, Ribeiro Vieira Lima, S. C. ${ }^{3}$, \\ Martinez-Romero, A. ${ }^{4}$, Frizzone, J. A. ${ }^{5}$ \\ ${ }^{1}$ Tecnóloga en riego, Estudiante de Doctorado, Centro Regional de Estudios del Agua \\ (CREA), UCLM, Ctra. de Las Peñas km. 3.2, 02071 Albacete, España; \\ anakelliane@gmail.com \\ ${ }^{2}$ Dr. Ingeniero Agrónomo, Catedrático de Universidad; Departamento de Producción Vegetal \\ y Tecnología Agraria, Universidad de Castilla-la Mancha, UCLM, España; \\ jose.tarjuelo@uclm.es \\ ${ }^{3}$ Investigador, Instituto de Pesquisa e Inovação na Agricultura Irrigada (INOVAGRI), Brasil \\ silviocarlos@inovagri.org.br \\ ${ }^{4}$ Dr. Ingeniero Agrónomo, Profesor Asociado; EIIAB, Departamento de Mecánica Aplicada e \\ Ingeniera de Proyectos, UCLM, España; angel.mromero@uclm.es \\ ${ }^{5}$ Dr. Ingeniero Agrónomo, Profesor Titular, Escola Superior de Agricultura Luiz de Queiroz- \\ ESALQ/ USP, Brasil frizzone@usp.br
}

\section{Resumen}

Un Servicio de Asesoramiento al regante (SAR) tiene como objetivo principal la optimización del uso del agua en la agricultura, dando respuesta a las demandas tecnológicas de los regantes. El objetivo del trabajo es evaluar la implantación y los beneficios de un modelo de SAR en Brasil, planteado para ofrecer una programación individualizada de riego en parcela a cada uno de los usuarios, basado en el uso de la herramienta on-line "Web S@l". La herramienta incluye en su base de datos una parte fija con información relativa a cada parcela (características del suelo, cultivo, caracterización del sistema de riego, etc ) y otra dinámica que incorpora, tanto la información obtenida de las estaciones agrometeorológicas en la zona que permite calcular la Evapotranspiración de referencia (ETo) diaria utilizando el método de Penman-Monteith, como los valores de los coeficientes de cultivo (Kc), obtenidos en base al seguimiento en campo de Iso técnicos. Las encuestas indican que el $31 \%$ de los agricultores siguen la información que se les facilita y que la productividad del agua (WP) aumenta en los usuarios del sistema S@l mejorando la gestión del agua en el Distrito de riego DIBAU.

\section{Introducción}

La eficiencia técnica de riego, que es la forma de hacer las mismas cosas, pero usando poca agua, es el preferido por los tomadores de decisiones, sobre el problema de la escasez de agua. Para evaluar el efecto de la eficiencia técnica en una sociedad dada, requiere un debate sobre la productividad del agua, que se puede expresar como agua de uso agrícola por unidad de volumen (Playan; Mateos, 2006).

Playán y Mateos (2006) encontraron que, en grandes y antiguos distritos de riego en España, se alentó a las técnicas de gestión para reducir el consumo excesivo de agua de determinados cultivos; Como resultado, se lograron ahorros considerables en agua, con casi los mismos rendimientos de los cultivos. Esto sólo con la orientación y la reeducación de los agricultores.

Un Servicio de Asesoramiento al regante (SAR) tiene como objetivo principal la optimización del uso del agua en la agricultura, dando respuesta a las demandas 
tecnológicas de los regantes en aspectos tales como la estimación de las necesidades de agua de los cultivos, la programación de riego, evaluar y analizar el funcionamiento de los sistemas de riego o planificar la formación de los regantes, tomando decisiones integradas con el agricultor.

A pesar de los esfuerzos significativos en muchas regiones del mundo, suele haber muchas limitaciones para la implementación de un Servicio de Asesoramiento de riego SAI, así como para su desarrollo presente y futuro. Estas limitaciones son de naturaleza muy diferente: política, económica y técnica. Algunas de ellas aparecen en el inicio del servicio y otros surgen en su propio desarrollo, lo que puede limitar su eficacia (Mateos, 2008).

Las primeras experiencias con servicios de asesoramiento al regante fueron realizadas en Estados Unidos (Eching 2002; English 2002), donde el CIMIS (California Irrigation Management Information System) puede tomarse como un referente que expone el potencial de los SAls. Aproximadamente $98 \%$ de la superficie regada en España se localiza en las Comunidades Autónoma que cuentan con SARs (3.526.197 ha). España, cuenta con una red de aproximadamente 500 estaciones agroclimáticas completas dentro de la red SIAR que son utilizadas por los 9 principales SARs. De entre ellos, el de Castilla-La Mancha, en funcionamiento desde el año 1999, ha colaborado directamente con más de 2000 agricultores, asesorando anualmente alrededor de 120000 ha de regadío.

EI Instituto INOVAGRI y el Instituto Nacional de Ciencia y Tecnología en Ingeniería de Riego (INCT-EI), con sede en ESALQ/USP, creado en 2011, planteó un proyecto con el objetivo de desarrollar un modelo de Servicio de Asesoramiento para el riego-SAl. El área piloto que sirvió como la observación y la experimentación fué el Distrito de Riego de Bajo Acaraú (DIBAU) en el estado de Ceará, Brasil.

El objetivo del trabajo es evaluar la implantación del Sistema de Asesoramiento al Regante (SAR) en Brasil, creado para planificar la programación de riego en parcela de cada uno de los usuarios, basado en el uso de la Web S@l como herramienta fundamental para la gestión del riego.

\section{Materiales y métodos}

El proyecto fue implementado en el Distrito de Riego Bajo Acaraú, con sede ubicada en el Triángulo de Marco, a $220 \mathrm{~km}$ de Fortaleza, en el norte del estado de Ceará. El clima es, de acuerdo con la clasificación de Thornthwaite y Mather (1955), Aw tropical lluvioso, lo que representa el clima cálido, con una precipitación media anual de $900 \mathrm{~mm}$, concentradas en los meses de enero a junio. Las temperaturas: mínimo, medio y máximo anual son 22.8 , 28.1 y $34.7^{\circ} \mathrm{C}$ respectivamente. La insolación de $2.650 \mathrm{~h}^{-1}$ año, la humedad relativa anual de $70 \%$, la velocidad del viento media de $3 \mathrm{~m} \mathrm{~s}^{-1}$ y la evaporación promedio anual de 1.600 $\mathrm{mm}$.

Para ejecutar el proyecto, era necesario conocer la situación actual del riego en la zona. Para esto se aplicó un formulario de catastro con el fin de conocer información sobre el productor y en su propiedad, el sistema de riego, detallando el equipo utilizado desde la bomba hasta el emisor, así como información sobre la gestión del riego, tipo de suelo, la plantación, las especies, variedades, etapa de crecimiento, la nutrición y la salud de los cultivos. Después de la preparación del formulario de registro comenzó la aplicación del cuestionario individual en todos los lotes.

\subsection{Evaluación de los Sistemas}

Después de conocer las características del perímetro de riego, se seleccionaron los agricultores que aceptaron participar del proyecto, fijándose 44 parcelas para realizar evaluaciones de Uniformidad de Emisión de Agua (UE) y la comprobación de posibles 
problemas durante la instalación y/o funcionamiento del sistema. Para determinar la UE, es necesario medir el caudal de los emisores a lo largo de las líneas laterales, y la presión de trabajo al comienzo de las líneas de derivación o unidad de trabajo. La UE se determinó mediante la ecuación 1, siguiendo la metodología de Keller \& Karmeli (1975), seleccionando 4 grupos de emisores en 4 laterales diferentes hasta conseguir los 16 grupos de emisores de control.

$$
U E=100 \frac{q_{25}}{q}
$$

Donde;

UE - uniformidad de Emisión (\%),

$\mathrm{q}_{25}$ - media de los $25 \%$ de los valores más bajos,

q - promedia de los valores.

\subsection{Sistema S@I}

En 2011 comenzó el desarrollo de software de gestión de riego. Un equipo formado por un analista de sistemas, un programador y un "diseñador de páginas web", junto con agrónomos y técnicos de riego del SAI, construyeron el nuevo software titulado Sistema de Asesoramiento al Regante - S@I. La base de datos se alimenta con: a) la información obtenida de una estación meteorológica automatizada del Instituto Nacional de Meteorología (INMET) que está instalada en el Distrito de Riego Bajo Acaraú (DIBAU) para calcular la ETo diaria utilizando el Penman-Monteith (Allen et al., 1998); b) los valores de los coeficientes de cultivo (Kc), obtenidos de investigaciones y estudios locales en la región norte, y en ausencia de ellos, se utilizan los datos de FAO (Allen et al., 1998); y c) las entrevistas a los regantes para conocer los datos de suelo, cultivo, caudal de emisores, etc. antes comentados.

A partir de esta información se, genera, para cada agricultor, una recomendación de tiempo de riego $(\mathrm{TI})$ según las características de su sistema de riego, con el fin de aportar la dosis de riego pretendida de acuerdo con el balance simplificado de agua en el suelo (Lira et al., 2011; Lima et al., 2012), y envía un SMS o correo electrónico al agricultor.

En 2012, tras realizar un conjunto de pruebas preliminares del sistema, se inició el envío de mensajes a los agricultores con la información antes comentada, y después de dos meses de funcionamiento del servicio se celebró una entrevista con 60 regantes seleccionados para evaluar el recibo, la receptividad y la adopción de la mensajes. El cuestionario tenía el siguiente contenido. 


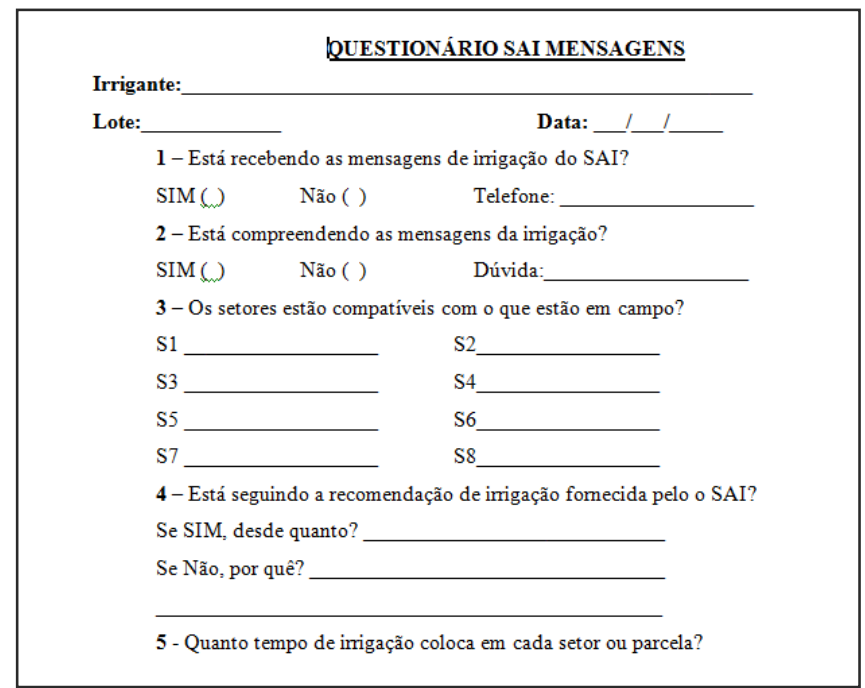

Figura 1. El modelo de formulario de registro aplicada a los regantes.

En 2013 un nuevo formulario fue distribuido en lo Perímetro de regadío para los usuarios del sistema, con el fin de conocer la aceptación del Proyecto SAI. Las preguntas dirigidas fueron las siguientes: a) ¿Está recibiendo, comprende y sigue la información de riego proporcionada por el SAI?; b) ¿Qué beneficios fueron identificados con el Proyecto SAI?; c)¿Quieres recibir o continuar recibiendo la información a través de SMS o correo electrónico?

También se ha evaluado, en tres períodos, los indicadores económicos ligados al SAI con el objetivo de analizar los beneficios del sistema. Para esta evaluación, se definió el indicador económico de la productividad del agua, con los datos de volumen de agua distribuida en el Distrito de riego DIBAU y los datos proporcionados por el sistema S@। como recomendaciones de riego para el Distrito, como se muestra en las ecuaciones 2 y 3.

Para la evaluación de productividad de agua realmente utilizada por DIBAU se definió la ecuación (2).

$$
\mathrm{WP}_{\text {DIBAU }}(€ \mathrm{~m}-3)=\frac{\text { Valoranualde la producción agrícola }}{\text { Volume anual de riego derivado al Distrito }}
$$

En la evaluación de la productividad del agua indicada por S@I, si el distrito usaría en ese momento, se definió la ecuación (3).

$$
\mathrm{WP}_{\mathrm{S} @ \mathrm{I}}(€ \mathrm{~m}-3)=\frac{\text { Valor anual de la producción agrícola }}{\text { La evapotranspiração del cultivo calculado por S@I }}
$$

\subsection{Resultados y discusión}

Este distrito cuenta con 8.335 hectáreas, divididas en 522 parcelas, con los agricultores organizados en una comunidad de regantes. Las parcelas son pequeñas (8 ha), medianas (16 a $18 \mathrm{ha}$ ) y grandes ( 80 a $200 \mathrm{ha}$ ), con cultivos frutales y hortalizas, destinadas al mercado interno y la exportación. Los datos recogidos muestran que la superficie total se 
divide entre los colonos, técnicos y empresarios en un total de 337 productores que se distribuyen como se muestra en la Tabla 1.

Tabla 1. Régimen de ocupación de DIBAU

\begin{tabular}{|c|c|c|}
\hline Discriminación & Regantes & Área (ha) \\
\hline Colonos & 310 & 2480 \\
\hline Técnicos & 13 & 235 \\
\hline Empresarios & 14 & 1127 \\
\hline Reserva legal & 1 & 1556 \\
\hline Total & $337^{*}$ & $3842^{*}$ \\
\hline
\end{tabular}

De este total, 107 productores han optado por abstenerse de participar en la investigación. Varios, por falta de una mejor explicación, porque creían que esto serviría para recaudar más tasas o impuestos en lugar de un beneficio para la comunidad. Por lo tanto, 230 regantes activos participaron del cuestionario. Al final de la evaluación de los datos obtenidos, se encontró que de los 556 productores registrado, 60,6\% están activos y $39,4 \%$ no son activos (bosque virgen o inactivo). El $60,6 \%$, productores activos (lo que supone 337 productores) tienen 3.842 hectáreas de superficie total, pero sólo el $65 \%$, lo que equivale a 2.497 ha, son de regadío.

El agua del río Acarau se bombea para luego ser repartida por gravedad a través de los canales. El sistema de riego predominante es el tipo localizado (goteo y microaspersión), sin embargo, hay un $3 \%$ de la superficie con riego por aspersión (Figura 2).

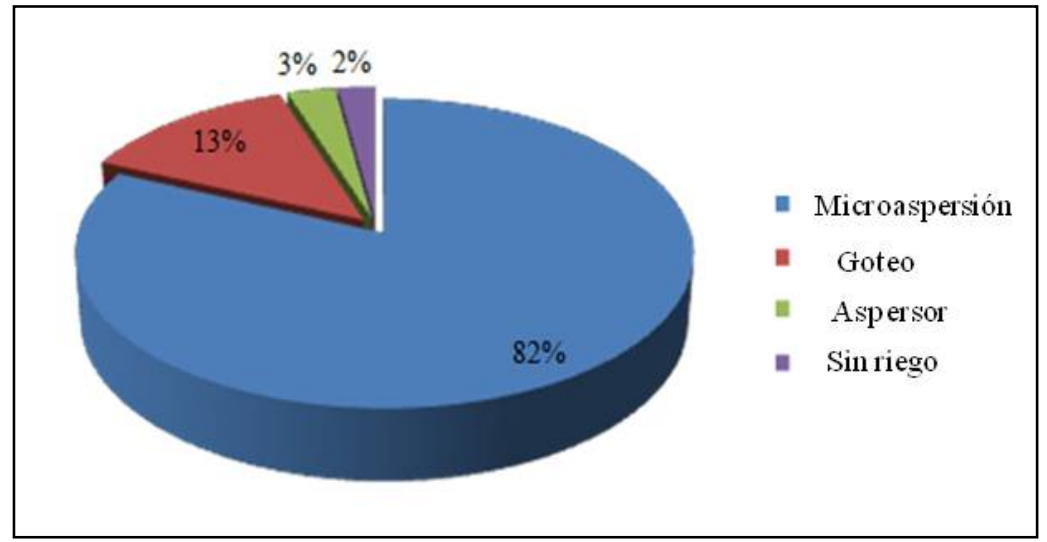

Figura 2. Distribución de sistemas de riego en el Perímetro de Regadío Bajo Acaraú.

En el perímetro de riego, sólo el 4,8\% de las zonas de regadío tenía un método de gestión del agua relativamente bien definido. En el resto de casos el regante sólo utiliza la intuición visual para definir se riega o no. El predominio del suelo es arenoso a franco arenoso y la principal actividad es el cultivo de frutas, sobre todo que la plantaciones de coco, plátano, guayaba y papaya.

\subsection{Evaluación de los sistemas de riego}

Tras la finalización de las evaluaciones de los productores seleccionados, se puede observar en la siguiente tabla los resultados. 
Tabla 2. Classificação de los sectores conforme UE sugerida por Bralts (1986).

\begin{tabular}{|l|c|c|}
\hline Clasificación & $\mathrm{UE}(\%)$ & $\mathrm{N}^{\circ}$ de Sectores \\
\hline Excelente & $90-95$ & 11 \\
\hline Bien & $80-89$ & 13 \\
\hline Aceptable & $70-79$ & 11 \\
\hline Malo & $<70$ & 9 \\
\hline
\end{tabular}

Los reultados en 11 sectores clasificados como aceptable se deben a un largo tiempo de uso con prácticas de gestión no muy adecuadas. En el caso de los 9 sectores clasificados como Malo, los problemas encontramos fueron fundamentalmente: la ausencia de filtros, el gran número de fugas, la ausencia de emisores y la falta de conocimiento operativo.

A los 9 sectores clasificados como malo, los técnicos de lo SAI hicieron algunas sugerencias como: 1) quitar obstrucción de los emisores con la apertura de los finales de línea; 2) limpieza del sistema de filtración; 3) la sustitución de los emisores con diferente caudal que los demás. De ellos, sólo 3 siguieron las recomendaciones y se llevó a cabo una nueva evaluación, resultando en una mejora en los valores de la UE, respectivamente, de $9 \%$; a $81 \%$, de $52 \%$ a $65 \%$ y $57 \%$ a $84 \%$; en el segundo sistema de evaluación, como se muestra en la Figura 3. Sólo las áreas 33 y 32, cambió su clasificación de Malo a Bien, siguiendo los criterios de Bralts (1986).

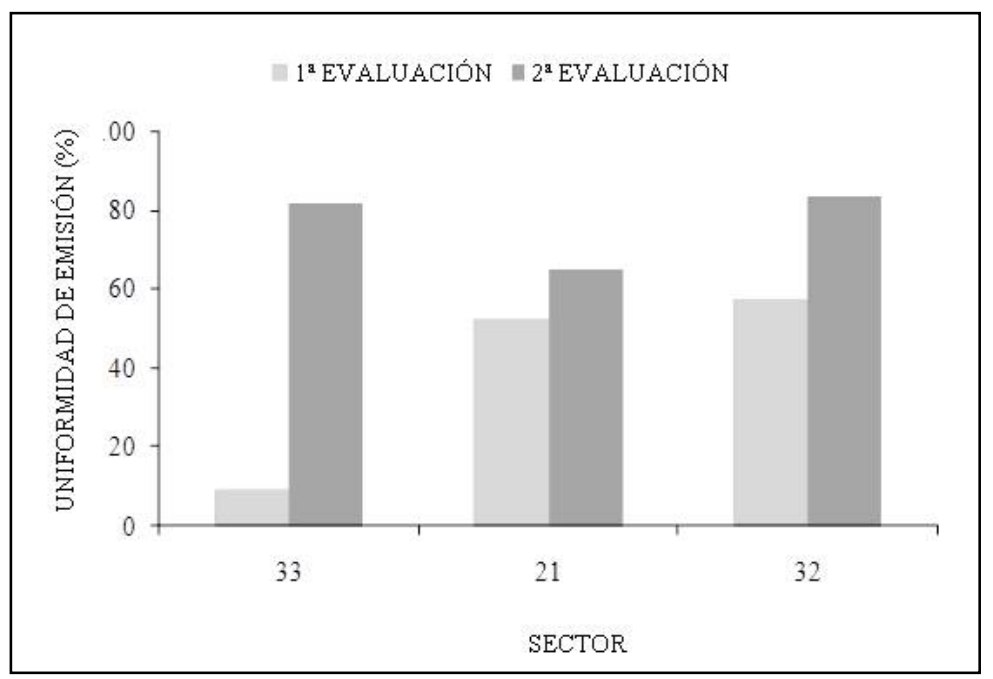

Figura 3. Uniformidad de agua en sectores reevaluado.

A los efectos de una gestión del riego correcto, la evaluación de la uniformidad es uno de los puntos básicos más importantes, y tiene como objetivo mejorar la productividad y/o la rentabilidad de la propiedad. En situaciones en las que el agua está limitada, tal como ocurre en muchas regiones del Brasil, el manejo eficiente tiene implicaciones importantes, ya que la falta de uniformidad del riego implica el exceso de agua en una parte del campo y su falta a otro, lo que lleva la necesidad de aumentar la profundidad del agua con el fin de lograr el mejor rendimiento (Mantovani et al., 2007).

\subsection{Envío de mensajes}

El 1 de agosto de 2012, se comenzó a enviar mensajes a través de SMS y el servicio web a los regantes. Los mensajes enviados contenían la información de Tiempo de Riego (TI). El número de mensajes se puede ver en la Figura 4. 


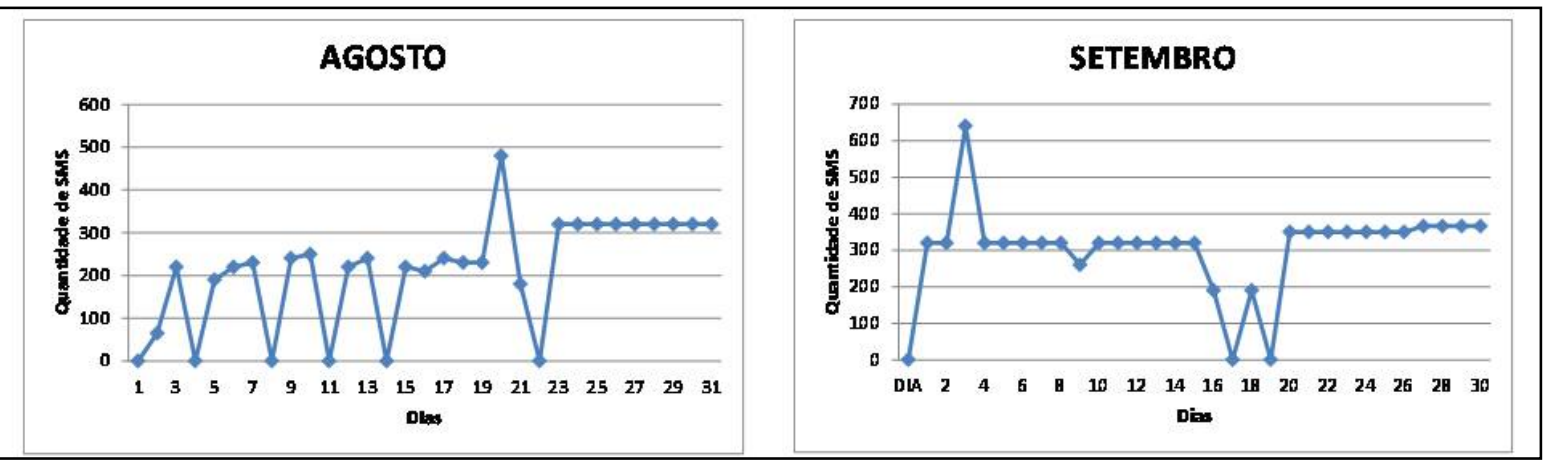

Figura 4. Los mensajes de texto enviados a los regantes de DIBAU en agosto y septiembre de 2012

En la Figura 5 se puede ver la receptividad de la información por los regantes al envío de mensajes por lo SAI.

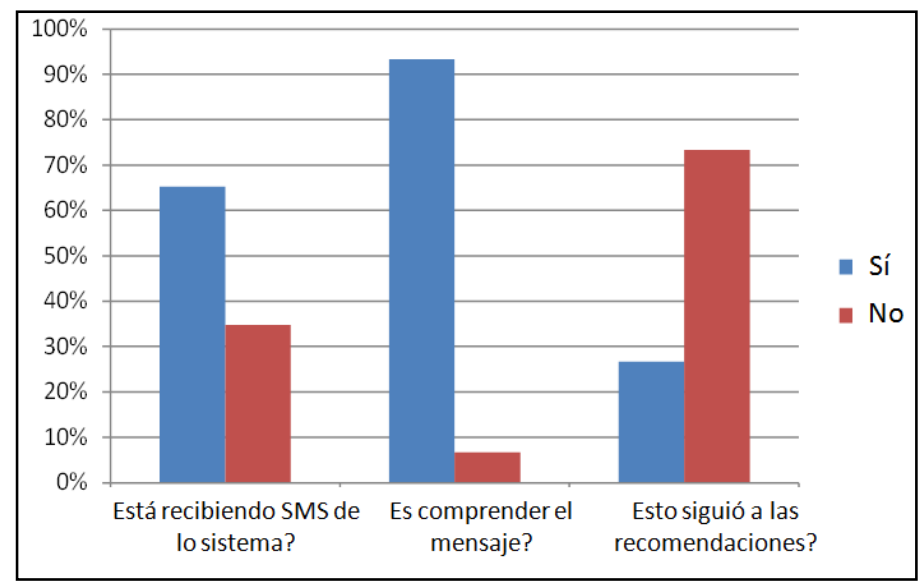

Figura 5. La receptividad por los regantes, de los SMS enviados por el SAI

Los resultados muestran que $65,22 \%$ estaban recibiendo y de éstos, $93,33 \%$ comprende el mensaje. La principal razón para no recibir, estaba en un error en el registro, pues por lo general el teléfono informado en registro no era el real. Entre las dudas más importantes de los regante está el no entender la nomenclatura, y algunos no entendían el propio sistema de envío de los mensajes.

Como consecuencia de ello, sólo el $27 \%$ utilizaron la información. Las principales razones para no seguir las recomendaciones fueron: a) no tener conocimiento de cómo adaptar el programador de riego; b) el regante creen que la recomendación está sobreestimando el requerimiento de agua del cultivo y c) existe incompatibilidad del tiempo total de riego con la disponiblidad de tiempo de riego al día.

En la investigación realizada en 2013, Sousa et al. (2013) encontraron que el número de regantes que recibieron la información suminsitrada por el SAR es de alrededor de $97 \%$ y algunos aspectos contribuyeron al logro de este número significativo, como la mejora de estructura de los informes, al hacerlos fáciles de entender y recibir la información a través de e-mail. Sin embargo, sólo el 36\% siguió las recomendaciones del SAR.

En la Tabla 3, dentro del conjunto de riegos presentado por Sousa et al. (2013), el resultado de la entrevista de este estudio mostraron que en comparación con los regantes que usan los tiempos de riego recibidos, aproximadamente el $31 \%$ lo siguen totalmente y el $41 \%$ solo en parte. 
Tabla 3. Forma de uso de los SMS recibidas

\begin{tabular}{|l|l|}
\hline FORMA DE USO DE LO TIEMPO DE RIEGO RECIBIDAS & $\%$ \\
\hline Siguén solo en parte & 41,02 \\
\hline Siguén totalmente & 30,77 \\
\hline Siguén siempre que sea posible & 28,21 \\
\hline
\end{tabular}

Entre los beneficios citados por los regantes que siguen las recomendaciones de los tiempos de riego enviados por el Proyecto SAI, se observó una mejora visual del $26 \%$ de los cultivos de regadío (Tabla 4 ).

Tabla 4. Beneficios de los Cultivos

\begin{tabular}{|l|l|}
\hline BENEFICIOS IDENTIFICADOS EN CULTURA & $\%$ \\
\hline Mejora en el aspecto visual & 25,64 \\
\hline No se nota ninguna alteración & 74,36 \\
\hline
\end{tabular}

La encuesta también encontró que los regantes quieren recibir la información, pero aproximadamente el $20 \%$ de los regantes están optando por recibir la información de tiempo de riego a través de correo electrónico. El muestreo mostró resultados similares a los reportados por Car et al. (2012). El autor al final de la temporada, realizó una entrevista con los usuarios y encontró que el $80 \%$ de los regantes desea utilizar el mensaje, pero no completamente, sino solo como un apoyo a la toma de decisiones, ya que otras características deben ser tomadas en cuenta, especialmente la apariencia visual de las plantas.

En la evaluación de la productividad, se analizaron los datos del agua repartida en Distrito de DIBAU (WP DIBAU) y los datos calculados por el sistema SAI (WP S@I). Para un análisis más detallado del proyecto se dividió en tres fases:

En la primera fase (julio-agosto/2012), se observó que en el Distrito (DIBAU) el consumo de agua, de manera efectiva para riego, fue $22.620 .408 \mathrm{~m}^{3}$, como registro total en los medidores de agua instalados en la zona de muchos agricultores. Este valor fue superior a la recomendada por el sistema S@I, que proporcionó una demanda de agua de los cultivos de $13.404 .788 \mathrm{~m}^{3}$ (59,26\% del total de agua medido como repartida). Si los agricultores hubieran utilizado la recomendación de S@I, el distrito tendría un ahorro de 9.215.620 $\mathrm{m}^{3}$ de agua.

En la segunda fase (agosto/2012-Jul/2013), el consumo del Distrito fue mayor (43.220.476 $\mathrm{m}^{3}$ ) que la recomendación del sistema S@। $\left(31.762 .753 \mathrm{~m}^{3}\right)$. Esta superioridad fue equivalente a $73.5 \%$ del volumen total recomendado por el programa. Si los regantes hubieran seguido la recomendación del S@I, el Distrito habría logrado un ahorro de 11.457.724 $\mathrm{m}^{3}$ de agua durante este período.

La aplicación de agua en la tercera etapa de este análisis fue $45.513 .148 \mathrm{~m}^{3}$, lo que demostró una vez más que está por encima del valor recomendado por S@। como necesidad de agua de los cultivos $\left(38.157 .401 \mathrm{~m}^{3}\right)$, equivalentes al $83,8 \%$ de agua aplicada, representando un ahorro de $7.355 .748 \mathrm{~m}^{3}$ de agua.

En la Figura 6 se puede ver la productividad del agua (WP) de DIBAU varió de $2,21 € \cdot \mathrm{m}^{-3}$ a $3,74 € \cdot \mathrm{m}^{-3}$ y WP del S@I varía desde $3.87 € \cdot \mathrm{m}^{-3}$ a $4,49 € . \mathrm{m}^{-3}$. En todas las situaciones, la WP S@l fue más alta que la WP DIBAU (Lima et al., 2015). 


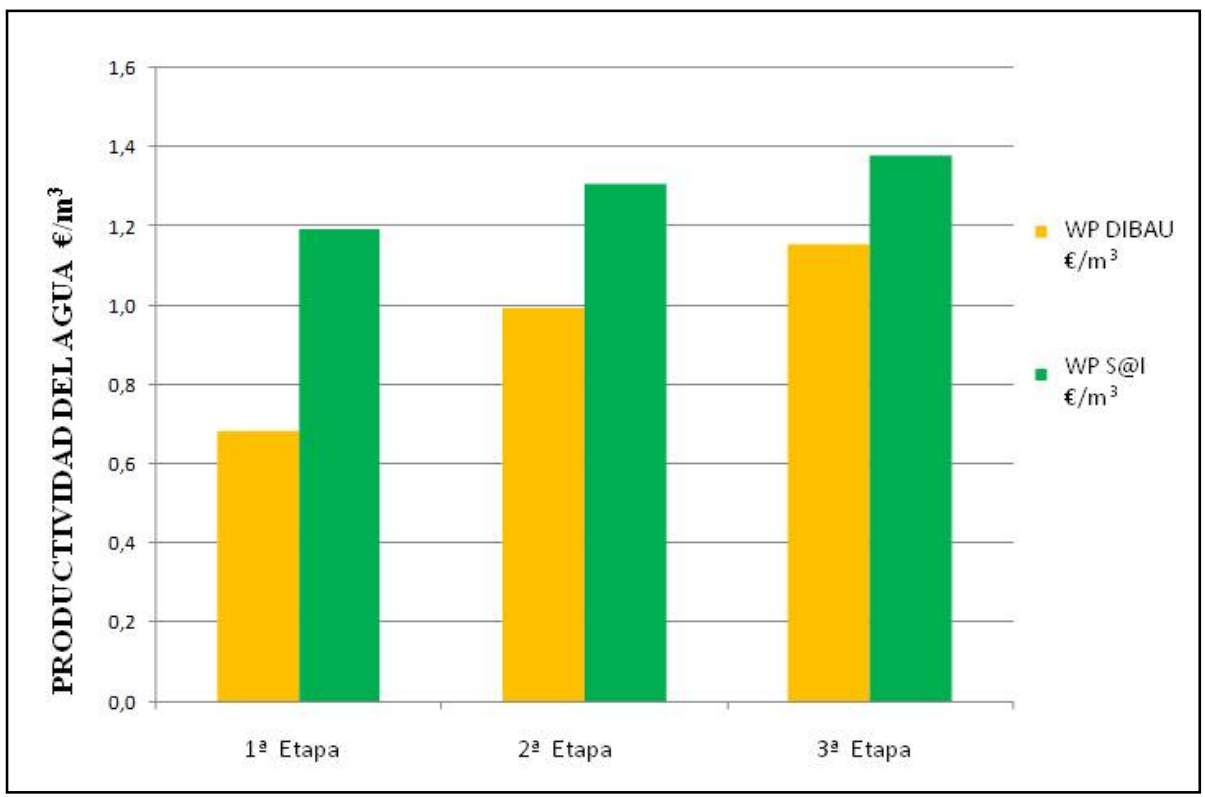

Figura 6. Resultados de la Productividad del Agua en DIBAU obtenidos por S@।

\subsection{Conclusiones y recomendaciones}

El sistema envía la información correcta y debe ser utilizada como una herramienta de gestión para el riego en las zonas de regadío;

El reto es mejorar aún más la comunicación con los agricultores para una transferencia de la información riego más eficiente y mejorar en la aceptación y seguimiento de las recomendaciones;

Con la evaluación de la productividad del agua (WP), el sistema S@। muestra que es eficiente a nivel general y demostró el posible beneficio que este sistema puede proporcionar la gestión del agua en el Distrito DIBAU.

\subsection{Agradecimientos}

Coordenação de Aperfeiçoamento de Pessoal de Nível Superior - CAPES.

\subsection{Bibliografía}

Allen RG, Pereira LS, Raes D, Smith M., Crop evapotranspiration: guidelines for computing crop water requirements. Irrigation and Drainage. Paper 56. FAO, Rome, Italy, 1998

Bralts VF. Field performance and evaluation. In: Nakayama FS, Bucks DA, (Ed.) Trickle irrigation for crop production. Amsterdam: Elsevier, 1986. p.216-240. (Development in Agricultural Engineering, 9).

CAR N. J., CHRISTEN E. W., HORNBUCKLE J. W., MOORE G. A. Using a mobile phone Short Messaging Service (SMS) for irrigation scheduling in Australia - Farmers' participation and utility evaluation, Computers and Electronics in Agriculture, 84, 132143, 2012 
ECHING S. Role of technology in irrigation advisory services: The CIMIS experience. 18th Congress and 53rd IEC meeting of the International Commission on Irrigation and Drainage (ICID). FAO/ICID International workshop on Irrigation Advisory Services and Participatory Extension Management. 24 July 2002. Montreal, Canada

ENGLISH M. Irrigation advisory services for optimum use of limited water. 18th Congress and 53rd IEC meeting of the International Commission on Irrigation and Drainage

(ICID). FAO/ICID International workshop on Irrigation Advisory Services and Participatory Extension Management. 24 July 2002. Montreal, Canada

Keller J, Karmeli D. Trickle irrigation design. Glendora: Rain Bird Sprinkler. 1975.133 p.

LIMA, S. C. R. V. ; SOUZA, F. ; FRIZZONE, J. A. ; CAMARGO, D. C. ; BELTRAO JUNIOR, J. A. ; NASCIMENTO, A. K. S. . Desempenho do sistema de assessoramento ao irrigante - S@। para a gestão da água em áreas irrigadas: benefícios aos irrigantes e ao distrito. Revista Brasileira de Agricultura Irrigada, v. 9, p. 1-13, 2015.

LIRA, J. V.; MOURÃO JÚNIOR, F.; SOUSA, A. E. C.; LIMA, S. C. R. V. ; FRIZZONE, J. A; VIANA, J. M. Auditoria de Desempenho de Sistemas de Irrigação I: A experiência inicial do Serviço de Assessoramento ao Irrigante - SAI, no Distrito de Irrigação do Baixo Acaraú. Revista Brasileira de Agricultura Irrigada, v.5, p. 265-271, 2011

Mantovani EC, Bernardo S, Palaretti LF. Irrigação - Princípios e Métodos. 2. ed. Viçosa: Editora UFV, 2007. v. 1. 358 p.

Mateos L, Identifying a new paradigm for assessing irrigation system performance Irrigation Science, Amsterdam, v. 27, p. 25-34, 2008.

PLAYÁN, E.; MATEOS, L. Modernization and optimization of irrigation systems to increase water productivity Agricultural Water Management, Amsterdam, v. 80, p. 100-116, 2006.

SOUSA, E. C.; SANTOS NETO, A. M.; LIMA, S. C. R. V.; FRIZZONE, J. A.; NASCIMENTO, A. K. S.; BELTRÃO JÚNIOR, J. A. Utilização da tecnologia da informação para o envio de mensagens via SMS ao irrigante: a experiência inicial do projeto SAI. Revista Brasileira de Agricultura Irrigada, v. 7, p. 224 - 234, 2013

THORNTHWAITE, C.W.; MATHER, J.R. The water balance. Climatology, Centerton, NJ. v.8, n.1. 1955. 